\title{
Empresas mais sustentáveis gerenciam menos os seus resultados? um estudo em empresas brasileiras de capital aberto
}

\section{More sustainable companies manage their results less? a study in brazilian public companies}

\author{
DANNIE DELANOY CARR QUIRÓS \\ Universidade Federal de Uberlândia \\ FERNANDA MACIEL PEIXOTO \\ Universidade Federal de Uberlândia \\ CLAUDIA OLÍMPIA NEVES MAMEDE MAESTRI \\ Universidade Federal de Uberlândia
}

\section{RESUMO}

A literatura aponta que as empresas com boas práticas de sustentabilidade apresentam melhores características financeiras e de mercado. A sustentabilidade tem sido amplamente adotada nas empresas ao redor do mundo para satisfazer o desejo dos acionistas por uma maior transparência (SIEW, 2015). Uma forma de se medir a transparência das firmas é por meio do gerenciamento de resultados (MARTINEZ, 2008). Segundo Bona-Sánchez, Pérez-Alemán e Santana-Martin (2017) existe uma relação positiva entre sustentabilidade e divulgação de informação contábil, o que faz com que empresas sustentáveis tendam a gerenciar menos os seus resultados (KIM; PARK; WIER, 2012). Considerando este contexto, o objetivo deste trabalho é estudar a relação entre a sustentabilidade empresarial e o gerenciamento de resultados. Para isto, foram desenvolvidos testes e regressões para empresas brasileiras de capital aberto no período de 2010 a 2017, utilizando diferentes formas de mensurar o gerenciamento de resultados. Os resultados da pesquisa indicam que não há uma relação entre sustentabilidade empresarial e gerenciamento de resultados. Po- 
rém foi encontrada uma relação significante entre gerenciamento de resultados e tamanho da firma.

Palavras-chaves: Sustentabilidade Empresarial, Gerenciamento de Resultados, Transparência.

\section{Abstract}

The literature points out that companies with good sustainability practices have better financial and market characteristics. Sustainability has been widely adopted in companies around the world to satisfy shareholders' desire for greater transparency (SIEW, 2015). One way to measure firm transparency is through earnings management (MARTINEZ, 2008). According to Bona-Sánchez, Pérez-Alemán and Santana-Martin (2017), there is a positive relationship between sustainability and disclosure of accounting information, which makes sustainable companies tend to manage their results less (KIM; PARK; WIER, 2012). Considering this context, the objective of this paper is to study the relationship between business sustainability and earnings management. For this, tests and regressions were developed for publicly traded Brazilian companies from 2010 to 2017, using different ways to measure earnings management. The results indicated that there is no relationship between business sustainability and earnings management. However, a significant relationship was found between earnings management and firm size.

Keywords: Corporate Sustainability, Earnings Management, Transparency.

\section{INTRODUÇÃo}

A literatura aponta que empresas mais sustentáveis tendem a apresentar maior desempenho, maior valor de mercado e menor risco. Além disso, empresas sustentáveis tendem a ter um menor custo da dívida e um menor endividamento (KHAN; SERAFEIM; YOON, 2015; LAMEIRA et al., 2013; PEIXOTO et al., 2016).

Ademais, empresas com boas práticas de sustentabilidade têm maiores possibilidades de demonstrar um papel mais ativo no mercado acionário, maior qualidade da informação financeira, melhores processos organizacionais e melhor desempenho financeiro (AMEER; OTHMAN, 2012; ECCLES; IOANNOU; SERAFEIM, 2014; KANTABUTRA, 2011; KHAN; SERAFEIM; YOON, 2015; WEBER, 2017). 
Segundo Siew (2015), a divulgação de sustentabilidade tem sido adotada amplamente por organizações ao redor do mundo para satisfazer o desejo dos acionistas por mais transparência em relação a aspectos ambientais e sociais.

De acordo com Martinez (2008) uma forma de se mensurar a transparência de uma organização é através do gerenciamento de resultados. O gerenciamento de resultados é um ato não ético que consiste em alterar a informação financeira para proteger ou satisfazer os interesses de um grupo da empresa mediante ocultação ou manipulação de informação como indica Leuz et al. (2003). As empresas gerenciam os seus resultados como uma reação aos estímulos do mercado. Alterar os resultados para evitar comunicar perdas, compensar o desempenho recente quando se tem prejuízo e piorar os resultados atuais para que os resultados no futuro pareçam melhores são alguns exemplos de gerenciamento de resultados.

De acordo com Caldas, Tambosi Filho e Vieira (2014) e Bona-Sánchez, Pérez-Alemán e Santana-Martin (2017), existe uma relação positiva entre sustentabilidade empresarial e a divulgação de resultados contábeis. Além disso, para Kim, Park e Wier (2012) as empresas sustentáveis tendem a gerenciar menos os seus resultados.

Martinez e Moraes (2017) explicam que há fatores que podem induzir ao gerenciamento de resultados, como mal remuneração dos serviços de auditoria e/ou quando existir alta dependência do conselho de administração. Conforme Lehmann (2016) as empresas com melhor governança corporativa tendem a ser mais agressivas para impedir o gerenciamento de resultados. Além disso, segundo Leuz et al. (2003), o gerenciamento de resultados tende a diminuir quando a proteção aos acionistas minoritários aumenta. Segundo os estudos de Rathke, Santana, Lourenço e Dalmácio (2016), na América Latina as organizações gerenciam mais os seus resultados do que nos países anglo-saxões e europeus.

Depois de conhecer estes antecedentes, buscou-se responder a seguinte pergunta: Há relação entre sustentabilidade empresarial e o gerenciamento de resultados? Para isto, foi definida a seguinte hipótese: As empresas pertencentes ao ISE (Índice de Sustentabilidade Empresarial) da B3 apresentam menor gerenciamento de resultados do que as demais empresas. O objetivo deste capitulo 
é estudar qual é a relação entre a sustentabilidade empresarial e o gerenciamento de resultados. Para alcançar este objetivo se utiliza como base metodológica os trabalhos de: Leuz, Nanda e Wysocki (2003), Guimarães, Peixoto e Carvalho (2017), Peixoto et al (2016), Nardi e Nakao (2009), Consoni, Calauto e Lima (2017), Silva (2017); Reis, Lamounier e Bressan (2015), Sprenger, Kronbauer e Costa (2017), Cunha e Piccoli (2017), Martinez e Soares (2017), Rathke et al (2016), Dechow, Ge e Schrand (2010), Barros, Soares e Lima (2013); Kang e Sivaramakrishnan (1995).

No que tange ao método, a presente pesquisa aplicou como modelo econométrico uma regressão de dados em painel e um teste de robustez, para identificar a possível relação ou associação entre sustentabilidade e gerenciamento de resultados de empresas listadas na B3 no período de 2010 a 2017.

De forma geral, a relação entre gerenciamento de resultados e sustentabilidade empresarial ainda não foi aprofundada, estando a concentração de pesquisas voltada para sustentabilidade e desempenho empresarial em países desenvolvidos (GOYAL; RAHMAN; KAZMI, 2013). Em vista disso, a relevância do presente estudo reside em abordar a relação entre sustentabilidade e GR como mecanismo de governança corporativa em mercados emergentes, no caso, o Brasil. Além disso, devido aos sistemas legais e políticos, existem diferenças entre os processos de governança corporativa a nível mundial, sendo que os problemas de governança não são solucionados simplesmente imitando as práticas ou mecanismos de outros países. Portanto, os modelos de governança corporativa devem ser criados e avaliados de acordo com cada caso individual, o que também se aplica ao mecanismo Gerenciamento de Resultados (BHASA, 2004).

\section{REFERENCIAL TEÓRICo}

Na presente seção são apresentados os conceitos e hipóteses que alicerçaram o presente estudo.

\subsection{Sustentabilidade empresarial e índice ISE}

No século XX intensificou-se a preocupação mundial com os danos que o homem vem causando ao meio ambiente. No mundo 
todo estão ocorrendo debates, discussões, pesquisas e congressos sobre o crescimento econômico e industrial desmedido, que não considera os impactos ambientais, fazendo com que o futuro do meio ambiente e da sociedade fique comprometido. Muitas medidas e estratégias sustentáveis tem sido propostas para minimizar os impactos ambientais. Neste contexto, a partir de 1987, o conceito de sustentabilidade emergiu no livro "Our Common Future" (também conhecido como "Brundtland Report"), que foi publicado pela Organização das Nações Unidas (ONU) através da Oxford University Press e elaborado pela World Comission on Environment and Development (WCED). Esta obra reuniu diferentes líderes mundiais para tratar a questão da sustentabilidade e do desenvolvimento sustentável. Nesta perspectiva, o conceito de Sustentabilidade foi concebido como a forma de desenvolvimento que satisfaz as necessidades presentes sem comprometer a satisfação das necessidades futuras da humanidade (WCED, 1987).

Elkington (2001) define a sustentabilidade de uma forma mais geral, tendo como base um tripé que envolve as frentes econômica, social e ambiental. Segundo ele, as empresas devem atribuir pesos equivalentes para cada uma dessas frentes. Ademais, o autor afirma que o lucro não deve ser visto como o único objetivo das empresas, mas sim sua missão, pois o lucro é condição para que a missão seja alcançada.

Em linha similar, Giovannoni e Fabietti (2013) oferecem um conceito multidimensional de sustentabilidade empresarial, que seria a integração de: (a) um sistema de governança para uma efetiva gestão do desempenho social, financeiro e ambiental; (b) um adequado modelo de negócios que considere as dimensões sociais, financeiras e ambientais; e (c) um sistema de medição e divulgação que permita um planejamento, monitoramento, comunicação e execução de todas as etapas de gestão.

$\mathrm{Na}$ presente pesquisa adotou-se como medida de sustentabilidade o Índice de Sustentabilidade Empresarial (ISE) da bolsa brasileira (B3), que também consiste numa medida multidimensional da sustentabilidade empresarial. A sustentabilidade segundo o ISE envolve sete dimensões: Natureza do Produto; Geral; Governança Corporativa; Social; Econômico-Financeira; Ambiental; e Mudanças 
Climáticas. Essas sete dimensões remetem ao tripé proposto por Elkington (2001). A dimensão geral está relacionada aos compromissos que as empresas possuem no que se refere à transparência e medidas anticorrupção. A dimensão de governança corporativa compreende características relacionadas à auditoria e fiscalização, conduta e conflito de interesses, conselho de administração e propriedade da empresa. As dimensões social, econômico-financeira e ambiental correspondem às políticas, gestão, desempenho e cumprimento legal nestes aspectos. Em relação à dimensão "mudanças climáticas", ela trata de políticas, gestão e reporte ambiental da empresa. A última dimensão corresponde à natureza do produto que trata dos impactos pessoais, impactos difusos e informação ao consumidor sobre os produtos e serviços que são comercializados pela companhia (B3, 2019).

O ISE foi criado em 2005 pela bolsa de valores brasileira. Originalmente ele foi financiado pela International Finance Corporation (IFC), braço financeiro do Banco Mundial, e sua metodologia é responsabilidade do FGVces e da B3. O ISE é considerado hoje uma iniciativa pioneira na América Latina que busca criar um ambiente de investimento compatível com as demandas de desenvolvimento sustentável da sociedade contemporânea e estimular a responsabilidade ética das organizações (B3, 2019).

Segundo dados da B3, a mais recente carteira do ISE foi anunciada em 29 de novembro de 2018 e vigora no período de 07 de janeiro de 2019 a 03 de janeiro de 2020. A nova carteira do índice reúne 35 ações de 30 empresas. Isso representa 14 setores econômicos e soma $\mathrm{R} \$ 1.880 .452 .652 .279,30$ em valor de mercado. Esse montante equivale a $48,06 \%$ do total do valor das companhias com ações negociadas na B3. Desde a sua criação, em 2005, o ISE apresentou rentabilidade de $+203,8 \%$ contra $+175,38 \%$ do Ibovespa. No mesmo período, o ISE teve também menor volatilidade: $24,67 \%$ em relação a $27,46 \%$ do Ibovespa (B3, 2019).

No que tange à metodologia do ISE, as empresas são escolhidas pelo Conselho Deliberativo do ISE (CISE) e devem atender aos seguintes critérios, conforme Tabela 1. 


\section{Tabela 1 - Critérios de inclusão e exclusão do ISE}

\begin{tabular}{ll}
\hline Critérios de & Descrição \\
\hline & 1. Ter presença em pregão de 50\% e na carteira de em- \\
& presas elegíveis nos últimos três anos. \\
& 2. Ser parte das primeiras 200 empresas segundo o índi- \\
ce de negociabilidade. & 3. Não ser classificada como Penny Stock. \\
& 4. Ser escolhida pelo CISE. \\
& 5. Cumprir com as exigências de sustentabilidade (re- \\
& presentadas pelas dimensões do ISE). \\
& 1. Quando uma empresa deixa de cumprir com os crité- \\
& rios de inclusão. \\
Exclusão & 2. Quando uma empresa estiver listada em uma situação \\
& 3. Quando o desempenho em sustentabilidade não for \\
satisfatório segundo o CISE.
\end{tabular}

Nota. Fonte: BM\&FBovespa (2015). Metodologia do Índice de Sustentabilidade Empresarial (ISE). São Paulo: BM\&FBovespa.

Para o critério de seleção, o ISE tem uma parceria com o Centro de Estudos em Sustentabilidade (GVces) da Escola de Administração de Empresas de São Paulo da Fundação Getúlio Vargas (FGV-EAESP). O GVces realizou um questionário como mecanismo de avaliação de sustentabilidade das empresas elegíveis. $\mathrm{O}$ índice conta com diversas dimensões relacionadas a meio ambiente, mudanças climáticas, econômico-financeira e social. Cada uma destas dimensões possui um grupo de critérios e, estes critérios possuem seus indicadores. É importante ressaltar que o preenchimento do questionário e a participação no índice é voluntário e representa o comprometimento da empresa com as questões da sustentabilidade (BM\&FBovespa, 2015). O Índice de Sustentabilidade (ISE) foi utilizado nesta pesquisa porque é uma ferramenta empregada na principal bolsa de valores brasileiras, além de ser elaborado em conjunto por mais de 10 (dez) instituições distintas entre elas: ABRAPP, IBRACON, ANBIMA, APIMEC, GIFE, IBGC, PNUMA, Instituto Ethos, Ministério do Meio Ambiente do Governo Federal e o Banco 
Mundial. Isto acrescenta robustez ao indicador, e também garante a transparente construção do indicador e seleção das empresas.

No que se refere aos trabalhos empíricos que adotam o ISE como medida de sustentabilidade, temos, por exemplo, o estudo de Lameira, Ness, Quelhas e Pereira (2013). No mercado de capitais brasileiro, melhores práticas de sustentabilidade estão relacionadas a melhor desempenho, maior valor e menor risco segundo Lameira et al. (2013). No período entre 2009 e 2013, empresas participantes do ISE apresentaram menor custo da dívida e menor endividamento como mostrado por Peixoto et al. (2016). Além disso, segundo Goyal, Rahman e Kazmi (2015), para empresas do setor da manufatura, o valor de mercado, a governança corporativa e a estratégia de negócios são características influentes no aumento do desempenho da sustentabilidade corporativa.

Segundo Chang e Kuo (2008), as empresas mais sustentáveis tendem a ter uma boa performance. Eccles et al. (2014) constataram que empresas estadunidenses que adotaram voluntariamente políticas de sustentabilidade mostraram procedimentos organizacionais mais eficientes que as empresas que não o fizeram, apresentando tendência de serem mais orientadas para o longo prazo e terem melhor desempenho contábil. Na República Checa as empresas estão conscientes da importância da sustentabilidade para atingir bom desempenho no longo prazo, sendo que a sustentabilidade deve ser parte integral da estratégia, do planejamento e do controle de acordo com Krechovská e Procházková (2014).

Segundo Rezaee e Tuo (2017), a divulgação de informação não financeira passada da firma (relatórios do ano anterior) está associada com o rendimento sustentável da empresa. Portanto, o rendimento sustentável atual pode estar ligado com a divulgação histórica de informação não financeira da empresa. De acordo com Ameer Othman (2012), Kantabutra (2011), Weber (2017) as empresas com maior sustentabilidade apresentaram um melhor desempenho financeiro medido pelo retorno sobre os ativos, e pelo lucro antes de impostos e fluxo de caixa das operações, em relação às empresas consideradas não sustentáveis.

Para a presente pesquisa foi utilizada uma variável categórica que assume o valor de 2 se a empresa pertence ao ISE, 1 se a em- 
presa esteve na lista de elegíveis, porém não foi selecionada para o índice final e 0 se não pertence ao ISE e nem estava na lista de elegíveis. Esta é uma proxy utilizada na maior parte das pesquisas para representar a sustentabilidade ou práticas sustentáveis. Na maior parte dos estudos, as características que tendem a se relacionar mais com o ISE são: tamanho da firma segundo Nunes, Teixeira, Nossa Galdi (2010) e rentabilidade da empresa de acordo com Martins, Campos e Martins (2016). Os investimentos sociais não explicam a adesão de empresas no ISE segundo os mesmos autores. Segundo Teixeira, Nossa e Funchal (2011), o fato de uma empresa participar do ISE pode fazer com que ela reduza suas possibilidades de financiamento externo e reduza seu risco, comparada com as empresas que não estão presentes no índice.

\subsection{Gerenciamento de resultados e sustentabilidade empre- sarial}

Nos últimos anos, a divulgação de sustentabilidade tem sido utilizada pelas firmas ao redor do mundo para satisfazer o desejo dos acionistas por mais transparência em relação a aspectos ambientais e sociais (SIEW, 2015).

De acordo com os estudos de Bona-Sánchez et al (2017), em empresas listadas na bolsa de valores da Espanha, no período de 2003 a 2012, encontrou-se uma relação positiva entre sustentabilidade e divulgação de resultados contábeis, que se fortalece na medida em que o acionista majoritário detém a propriedade sobre os fluxos de caixa. Isto porque, segundo o estudo, o acionista controlador se esforça para promover relações no longo prazo com os outros acionistas por meio da divulgação da sustentabilidade como forma de melhorar a reputação da firma e manter a credibilidade financeira no mercado.

Sob a mesma ótica, em um estudo sobre os relatórios de sustentabilidade de empresas da Malásia no período entre 2011 e 2013 foi demonstrado que o desempenho financeiro e o tamanho da companhia tendem a ter uma relação positiva com a qualidade dos relatórios de sustentabilidade, porém os accruals anormais e a alavancagem não revelaram relação significante com a qualidade destes relatórios de sustentabilidade (IBRAHIM et al., 2015). 
De acordo com Yip et al. (2011), há uma relação negativa entre a divulgação da responsabilidade social corporativa e o gerenciamento de resultados. Na indústria do óleo e gás foi achada esta relação negativa entre responsabilidade social corporativa e gerenciamento de resultados, porém, na indústria de alimentos encontrou-se uma relação positiva. Isso sinaliza que a relação entre responsabilidade social corporativa e gerenciamento de resultados é mais afetada pelo ambiente político do que pelas considerações éticas.

Segundo Chih et al. (2008) em um estudo que envolveu mais de 1600 empresas de 46 diferentes países ao redor do mundo se constatou que aparentemente empresas que tem um compromisso maior com a responsabilidade social corporativa mitigam o gerenciamento de resultados. Para 27 empresas listadas na bolsa de valores da Indonésia não existe influência entre gerenciamento de resultado e as práticas de responsabilidade social corporativa e, baseados também na responsabilidade social corporativa, as atividades associadas ao gerenciamento de resultados afetam negativamente o rendimento da companhia à longo prazo conforme Rahmawati e Dianita (2011).

Curiosamente a responsabilidade social empresarial pode ser uma ferramenta poderosa para os administradores de empresas que gerenciam resultados para ganhar apoio dos acionistas e assim reduzir significativamente as chances deles serem demitidos ou proteger outros interesses. A relação positiva entre responsabilidade social corporativa e rendimento financeiro é moderada quando é combinada com o gerenciamento de resultados de acordo com Prior, Surroca e Tribó (2007).

Considerando que as empresas sustentáveis têm menos probabilidade de gerenciar resultados como exposto por Kim et al. (2012), o presente trabalho definiu a seguinte hipótese de pesquisa: Há uma relação inversa entre gerenciamento de resultados e sustentabilidade empresarial em empresas brasileiras pertencentes ao ISE. Portanto, se espera que empresas classificadas como sustentáveis (presentes no ISE) gerenciem menos seus resultados.

\section{Metodologia}

A seguir são apresentadas as principais características metodológicas do trabalho que tem uma abordagem quantitativa do tipo descritiva. 


\subsection{Amostra}

A amostra do trabalho está composta por empresas brasileiras de capital aberto listadas na B3, com exclusão das empresas financeiras devido às suas particularidades econômicas e contábeis. Os dados foram coletados na base de dados Economatica, envolvendo o período de 2010 até 2017. O período inicial se deve ao fato de que em 2010 ocorreu a adoção dos International Financial Reporting Standards (IFRS) (Padrões internacionais de contabilidade) pelas empresas brasileiras segundo a Comissão de Valores Mobiliários (CVM) e o período final devido à disponibilidade dos dados no momento de execução da pesquisa. Foram eliminadas as empresas que não apresentaram nenhum valor em nenhuma variável durante o período em estudo. Depois de realizados os filtros e a seleção da amostra, esta ficou composta por 673 empresas.

\subsection{Variáveis Dependentes}

O gerenciamento de resultados foi a variável dependente. Foram adotadas duas métricas para medir o gerenciamento de resultados: o método de Leuz, Nanda e Wysocki (2003) e o método de Kang e Sivaramakrishnan (1995), que foi apresentado sob a forma de um teste de robustez.

No que se refere ao método de Leuz, Nanda e Wysocki (2003), a proxy adotada envolveu aquelas que tratam dos componentes contábeis (acumulações ou accruals em inglês). Foi utilizado este modelo por ser referência no cálculo do gerenciamento de resultados como utilizado por Holanda, Carlos e Coelho (2013) e Sandrin e Soares (2012). A variável gerenciamento de resultados é dividida em 4 "sub-variáveis", todas envolvendo a suavização de dados contábeis e de caixa. Para o cálculo destas variáveis é preciso mensurar os lucros após o imposto de renda (LDIR) e o fluxo de caixa operacional (CFO - cash flow from operations) respectivamente por meio das fórmulas 1 e 2:

LDIR = LAIR - Provisão IR + Reversão de Juros Sobre o Capital Proprio

$$
\mathrm{CFO}=\mathrm{LDIR}-\mathrm{ACC}(2)
$$


O ACC que se apresenta na fórmula 2 corresponde às acumulações ou accruals. Esta variável corresponde a itens contábeis que não representam entradas e saídas efetivas de caixa. O cálculo dos ACC é realizado por meio da fórmula 3:

$$
\mathrm{ACC}=(\Delta \mathrm{AC}-\Delta \mathrm{CASH})-(\Delta \mathrm{PC}-\Delta \mathrm{DC}-\Delta \mathrm{TP})-\mathrm{DEP}
$$

Onde:

$\Delta$ = Variação;

ACC $=$ Accruals

$\mathrm{AC}=$ Total de Ativo Circulante

$\mathrm{CASH}=$ Disponível e investimentos de curto prazo

$\mathrm{PC}=$ Total do Passivo Circulante

$\mathrm{DC}=$ Dívida de Curto Prazo

$\mathrm{TP}=$ Imposto de Renda a pagar

DEP $=$ Despesas de Depreciação e Amortização

A variável gerenciamento 1 (GRI) é calculada por meio do desvio padrão dos lucros operacionais (LDIR) dividido pelo desvio padrão do fluxo de caixa operacional (CFO) A variável de gerenciamento 2 (GIRII) é a correlação entre os accruals e o fluxo de caixa operacional. A variável de gerenciamento 3 (GRIII) é calculada pelo valor absoluto da divisão entre ACC e o CFO. Finalmente, a variável de gerenciamento 4 (GRIV) é um resumo geral das variáveis porque é a média simples de GRI, GRII e GRIII, e para tal variável, um maior valor demonstra um maior gerenciamento de resultados de acordo com Leuz et al. (2003). As quatro variáveis de gerenciamento (GRI, GRII, GRIII e GRIV) foram testadas, e após os testes a variável GRIV foi a que apresentou o melhor ajuste, e foi, portanto, utilizada.

Em relação ao modelo Kang e Sivaramakrishnan (1995), este está composto por duas etapas. Na primeira etapa são calculados os accruals totais e depois os accruals discricionários por meio de regressões utilizando variáveis instrumentais.

Para o cálculo dos accruals totais correspondentes à primeira etapa é utilizada seguinte equação:

$$
\operatorname{ATS}_{i t}=\beta_{0}+\beta_{1}\left[\delta_{1} \operatorname{REC}_{i t}\right]+\beta_{2}\left[\delta_{2} \mathrm{DES}_{\mathrm{it}}\right]+\beta_{3}\left[\delta_{3} \mathrm{ATP}_{\mathrm{it}}\right]+\varepsilon_{\mathrm{it}}
$$


Onde:

$$
\begin{aligned}
& \text { ATS }_{\text {it }}=\left(\triangle \mathrm{CGL}-\mathrm{DEP}_{\mathrm{it}}\right) / \mathrm{ATT}_{\mathrm{it}-1} \\
& \delta 1=\mathrm{CRR}_{{ }_{\mathrm{i}, \mathrm{t}-1}-1} / \mathrm{REC}_{\mathrm{i}, \mathrm{t}-1} \\
& \delta 2=\left(\mathrm{CGL}_{-} \mathrm{CRR}_{\mathrm{r}_{\mathrm{i}, \mathrm{t}-1}}\right) / \mathrm{DES}_{\mathrm{i}, \mathrm{t}-1} \\
& \delta 3=\mathrm{DEP}_{{ }_{\mathrm{i}, \mathrm{t}-1}-1} / \mathrm{ATP}_{\mathrm{i}, \mathrm{t}-1}
\end{aligned}
$$

ATS accruals totais da empresa;

REC = Receita Líquida da empresa e excluindo tributação;

DES = Custos e Despesas Operacionais antes da Depreciação e Amortização da empresa;

CGL = Capital de Giro Líquido da empresa, excluindo as disponibilidades, financiamentos de curto prazo e provisão de Impostos a Pagar;

$\mathrm{ATP}_{\text {it }}=$ Ativo permanente (Imobilizado + Diferido + Intangível+ Investimentos) da empresa;

ATT $=$ Ativo Total

$\mathrm{CRR}_{\mathrm{it}-1}=$ Contas a Receber da empresa;

$\mathrm{DEP}=$ Despesas de depreciação da empresa.

$\mathrm{i}=$ indicador cross-section;

$\mathrm{t}=$ indicador de tempo;

$\beta=$ coeficientes associados às variáveis independentes e de controle;

$\varepsilon=$ termo de erro da equação.

As variáveis $\mathrm{REC}_{\mathrm{it}{ }^{\prime}} \mathrm{DES}_{\mathrm{t}}$ e ATP foram escaladas em termos de ativos totais do período anterior. Os parâmetros $\delta 1$, $\delta 2$ e $\delta 3$ representam os indicadores de rotatividade que controlam pelas características específicas das empresas segundo Kang (1999). As variáveis instrumentais adotadas no modelo foram os indicadores de rotatividade como realizado por Martinez (2008) e Silva (2013).

Os accruals discricionários são os erros da equação $(\varepsilon)$ obtidos pela diferença entre os accruals totais e os accruals estimados como mostra a equação a seguir segundo Kang e Sivaramakrishnan (1995):

$$
\mathrm{ACD}_{\mathrm{it}}=\mathrm{ATS}_{\mathrm{it}}-\left\{\beta_{0}+\beta_{1}\left[\delta_{1} \operatorname{REC}_{\mathrm{it}}\right]+\beta_{2}\left[\delta_{2} \mathrm{DES}_{\mathrm{it}}\right]+\beta_{3}\left[\delta_{3} \mathrm{ATP}_{\mathrm{it}}\right]\right\}
$$

Onde:

$\mathrm{ACD}=$ Accruals discricionários da empresa 
Portanto, a presente pesquisa teve duas variáveis dependentes principais - uma seguindo a métrica de Leuz et al (2003) e outra que adotou o modelo KS (1995).

\subsection{Variável Independente}

A variável independente (ISE) utilizada na pesquisa é a sustentabilidade empresarial mensurada por meio da seguinte classificação: 0 para empresas que não pertencem ao ISE e que não estiveram presentes na lista de empresas elegíveis para fazer parte do ISE (Empresas não sustentáveis), 1 para empresas que estiveram presentes na lista de empresas elegíveis para comporem o ISE, mas ao final não conseguiram ser selecionadas para pertencer ao ISE (Empresas "quase sustentáveis") e 2 para as empresas que pertencem ao ISE (Empresas sustentáveis). Esta variável se fundamentou em Barros, Soares e Lima (2013); Cunha e Piccoli (2017); Dechow, Ge e Schrand (2010); Silva ( 2017). Espera-se uma relação negativa com o gerenciamento de resultados, ou seja, empresas participantes do ISE teriam menor probabilidade de gerenciar seus resultados.

\subsection{Variáveis de Controle}

Entre as variáveis de controle se encontra a Governança Corporativa (IGC) que foi utilizada nos trabalhos de Barros, Soares e Lima (2013), Cunha e Piccoli (2017), Dechow, Ge e Schrand (2010) e Silva (2017). É uma variável dummy que tem o valor de 1 quando a empresa é classificada no segmento de Novo Mercado e 0 quando não for classificada neste segmento. Se espera, para esta variável, uma relação negativa com o GR, isto é, se supõe que empresas pertencentes aos níveis de governança da bolsa gerenciem menos seus resultados contábeis. A variável ISE se diferencia do IGC porque ela mensura outras dimensões que não são atendidas pelo IGC como as dimensões ambientais, mudanças climáticas, natureza dos produtos e aspectos sociais (B3, 2019).

A variável Rentabilidade (ROA) corresponde ao retorno sobre o ativo total e é calculada pela divisão entre lucro líquido e o ativo total e é uma variável muito utilizada na literatura como fator influenciador de gerenciamento de resultados. Esta variável foi utilizada por Barros, Soares e Lima (2013), Consoni, Colauto 
e Lima (2017), Dechow, Ge, Schrand (2010), Martinez e Moraes (2017), Nardi, e Nakao (2009), Rathke et al (2016), Reis, Lamounier e Bessan (2015), Sprenger, Kronbauer e Costa (2017). No caso desta variável, os estudos divergem no tipo de relação com gerenciamento de resultados.

O Tamanho (TAM) corresponde à dimensão da empresa e é calculado pelo logaritmo natural dos ativos totais, sendo uma variável muito utilizada para explicar o gerenciamento de resultados utilizada nas pesquisas de Barros, Soares e Lima (2013), Consoni, Colauto e Lima (2017), Cunha e Piccoli (2017), Dechow, Ge e Schrand (2010), Nardi e Nakao (2009), Rathke et al (2016), Reis, Lamounier e Bessan (2015), Silva (2017) e Sprenger, Kronbauer e Costa (2017).O sinal da relação entre TAM e GR é incerto, pois há estudos que encontram ambos os sinais.

O Endividamento (END) é calculado pela divisão entre a dívida de longo prazo e o patrimônio líquido. Esta variável está presente nas pesquisas de Dechow, GE e Schrand (2010), Nardi e Nakao (2009), Silva (2017) e Sprenger, Kronbauer e Costa (2017). Se espera que quanto mais endividada se encontrar uma firma, maior será o seu gerenciamento de resultados. Por outro lado, a Concentração Acionária $(\mathrm{CON})$ é a porcentagem de ações ordinárias em poder do acionista majoritário. Segundo a literatura, quanto maior for a concentração acionária, maior será também o gerenciamento de resultados. Isto porque quando há uma alta concentração acionária, gerenciar resultados é mais fácil. Para fundamentar o uso desta variável no presente estudo foram usados os trabalhos de Consoni, Colauto, Lima (2017), Dechow, Ge e Schrand (2010) e Silva (2017).

Outra variável que afeta o gerenciamento de resultados, de forma negativa, são os Resultados Negativos (RES), segundo Cuadro-Ballesteros, Martínez-Ferrero, García-Sánchez (2017), Nardi, Nakao (2009); Rathke et al (2016), Sprenger, Kronbauger e Costa (2017). Esta variável é representada por meio de uma dummy que assume o valor de 0 quando o lucro líquido é zero ou maior que zero (não há prejuízo) e o valor de 1 quando o lucro líquido é menor do que zero (há prejuízo), se espera que tenha relação negativa com o gerenciamento de resultados indicando que quando o período apresenta prejuízo, os administradores vão gerenciar os resultados por 
diversas razões como melhorar a imagem da sua gestão ou manter benefícios e/ou privilégios. A variável Market-to-book (MTB) é a razão entre o valor de mercado da empresa e seu valor patrimonial e se aguarda que tenha uma relação negativa com o gerenciamento de resultados segundo Reis, Lamounier e Bressan (2015). Isto quer dizer que quando a empresa for mais valiosa, ela vai tender a gerenciar menos os resultados, provavelmente porque uma empresa com maior valor não tem que manipular os seus resultados para ter uma boa imagem ante o mercado e outsiders.

Finalmente, foi utilizada a variável Setor (SET) onde setor de cada empresa foi classificado em setor primário, secundário e terciário. Embora não se tenha como referência uma pesquisa com esta classificação para setor, se fundamenta o uso desta variável nos trabalhos de Cunha e Piccoli (2017), Rathke et al (2016), Silva (2017) e Sprenger, Kronbauer e Costa (2017). Em relação ao procedimento de separação dos setores foram consideradas como empresas do setor primário as empresas pertencentes aos setores de Agro e pesca, minerais não metálicos, mineração, petróleo e gás. Para o setor secundário foram considerados os setores de Alimentos e bebidas, eletrônicos, energia elétrica, máquinas industriais, papel e celulose, química, Siderurgia e metalurgia, têxtil, veículos e peças. Para o setor terciário, os setores de comércio, construção, outros, software e dados, telecomunicações, transporte e serviços.

Na Tabela 2 a seguir estão expostas as variáveis que compuseram a presente pesquisa.

\subsection{Modelos Econométricos}

Após a definição das variáveis do modelo econométrico, foram realizados testes para selecionar o tipo de modelo de dados em painel mais adequado aos dados e testes para diagnosticar problemas de autocorrelação, multicolinearidade e heterocedasticidade. Todos os processos foram feitos através do software estatístico Stata 13 @. O modelo utilizado se apresenta a seguir:

$$
\begin{gathered}
\mathrm{GRIV}=\beta_{0}+\beta_{1} \mathrm{ISE}_{\mathrm{it}}+\beta_{2} \mathrm{IGC}_{\mathrm{it}}+\beta_{3} \mathrm{ROA}_{\mathrm{it}}+\beta_{4} \mathrm{TAM}_{\mathrm{it}}+\beta_{5} \mathrm{END}_{\mathrm{it}}+\beta_{6} \mathrm{CON}_{\mathrm{it}} \\
+\beta_{7} \mathrm{RES}_{\mathrm{it}}+\beta_{8} \mathrm{MTB}_{\mathrm{it}}+\beta_{9} \mathrm{SET}_{\mathrm{it}}+\varepsilon_{\mathrm{it}}
\end{gathered}
$$




\section{Tabela 2 - Variáveis da pesquisa}

\begin{tabular}{|c|c|c|c|}
\hline Variável & Mensuração & $\begin{array}{l}\text { Sinal } \\
\text { Esperado }\end{array}$ & Referência \\
\hline $\begin{array}{l}\text { Variáveis dependentes } \\
\text { - Gerenciamento de } \\
\text { resultados (GRIV) - } \\
\text { Leuz } \\
\text { - Gerenciamento de } \\
\text { resultados (GRIV) } \\
\text { utilizada no Teste de } \\
\text { Robustez - KS }\end{array}$ & $\begin{array}{c}\text { GRIV }=\frac{G R I+G R I I+G R I I I}{3} \\
\operatorname{GRIV}_{\mathrm{it}}=\mathrm{ATT}_{\mathrm{it}}-\left\{\beta_{0}+\beta_{1}\left[\delta_{1} \mathrm{REC}_{\mathrm{it}}\right]+\beta_{2}\left[\delta_{2} \mathrm{DES}_{\mathrm{it}}\right]\right. \\
\left.+\beta_{3}\left[\delta_{3} \mathrm{ATP}_{\mathrm{it}}\right]\right\}\end{array}$ & & (14) \\
\hline $\begin{array}{l}\text { Variável Independente } \\
\text { Sustentabilidade } \\
\text { (ISE) }\end{array}$ & $\begin{array}{l}\text { Classificação de } 0 \text { para as não sustentáveis, } 1 \text { para as } \\
\text { quase sustentáveis e } 2 \text { para as sustentáveis. }\end{array}$ & $(-)$ & (2) e (3) \\
\hline $\begin{array}{l}\text { Variáveis de Controle } \\
\text { Governança } \\
\text { Corporativa (IGC) }\end{array}$ & $\begin{array}{l}\text { Variável dummy igual a } 1 \text { para as empresas } \\
\text { classificadas no Novo Mercado e valor } 0 \text { para as que } \\
\text { não estão neste segmento. }\end{array}$ & $(-)$ & $\begin{array}{l}(6), \quad(9) \\
(12),(13)\end{array}$ \\
\hline $\begin{array}{l}\text { Retorno sobre ativo } \\
\text { total (ROA) }\end{array}$ & $R O A=\frac{\text { Lucro Líquido }}{\text { Ativo Total }}$ & $(+/-)$ & $\begin{array}{l}\text { (4) (5), }(7), \\
(8) \\
(11), \quad(12),\end{array}$ \\
\hline $\begin{array}{l}\text { Tamanho da } \\
\text { empresa (TAM) }\end{array}$ & $T A M=\operatorname{Ln}($ Ativo Total $)$ & $(+/-)$ & $\begin{array}{l}\text { (13) } \\
\text { (4), (5), (6), } \\
(7),(8),(9), \\
(11), \quad(12), \\
(13)\end{array}$ \\
\hline $\begin{array}{l}\text { Endividamento } \\
\text { (END) }\end{array}$ & $E N D=\frac{\text { Dívida Longo Prazo }}{\text { Patrimônio Líquido }}$ & $(+)$ & $\begin{array}{l}(4),(6),(8), \\
(12)\end{array}$ \\
\hline $\begin{array}{l}\text { Concentração } \\
\text { acionária (CON) }\end{array}$ & $\begin{array}{l}\text { Porcentagem de ações ordinárias possuídas pelo maior } \\
\text { acionista. }\end{array}$ & $(+)$ & (5),(6), (12) \\
\hline $\begin{array}{l}\text { Resultados } \\
\text { Negativos (RES) }\end{array}$ & $\begin{array}{l}\text { Dummy } 1 \text { para ano com prejuízo e } 0 \text { para ano sem } \\
\text { prejuízo }\end{array}$ & $(+)$ & $\begin{array}{l}(4),(8),(10) \\
(11)\end{array}$ \\
\hline $\begin{array}{l}\text { Market-to-book } \\
\text { (MTB) }\end{array}$ & $M T B=\frac{\text { Valor de Mercado }}{\text { Patrimônio Liquido }}$ & $(-)$ & (7),(11) \\
\hline Setor (SET) & $\begin{array}{l}\text { Os setores da base Economática foram reclassificados } \\
\text { em setor primário, setor secundário e setor terciário. }\end{array}$ & $\mathrm{n} / \mathrm{a}$ & $\begin{array}{l}(6),(8),(9), \\
(11)\end{array}$ \\
\hline
\end{tabular}

Notas: (1) (LEUZ; NANDA; WYSOCKI, 2003); (2) (GUIMARÃES; PEIXOTO; CARVALHO, 2017); (3)(PEIXOTO et al., 2016) ; (4)(NARDI; NAKAO, 2009); (5) (CONSONI; COLAUTO; LIMA, 2017); (6) (SILVA, 2017); (7) (REIS; LAMOUNIER; BESSAN, 2015) ; (8) (SPRENGER; KRONBAUER; COSTA, 2017); (9)(CUNHA; PICCOLI, 2017); (10) (MARTINEZ; MORAES, 2017); (11) (RATHKE et al., 2016); (12) (DECHOW; GE; SCHRAND, 2010); (13) (BARROS; SOARES; LIMA, 2013); (14) (KANG; SIVARAMAKRISHNAN, 1995) Fonte: Elaboração própria.

\section{Onde:}

$\mathrm{i}=$ indicador cross-section;

$\mathrm{t}=$ indicador de tempo;

$\beta=$ coeficientes associados às variáveis independentes e de controle;

$\varepsilon=$ termo de erro da equação. 


\section{RESULTADOS}

A presente seção mostra os principais resultados da pesquisa depois do tratamento e análise dos dados coletados, envolvendo análises descritivas, correlações e regressões com dados em painel feitas através do software estatístico Stata 13 ®. Além disso, é feita uma análise dos principais achados em relação aos achados da bibliografia consultada.

\subsection{Análise Descritiva}

A Tabela 3 a seguir apresenta um resumo das principais estatísticas descritivas aplicadas a cada uma das variáveis do estudo como: observações, médias, desvio padrão, valores mínimos e máximos. A Tabela 3 apresenta a informação das empresas não sustentáveis (Não estão presentes no ISE), das empresas "quase sustentáveis" (Não estão presentes no ISE, mas compõem a lista das empresas elegíveis) e das empresas sustentáveis (Presentes no ISE).

Esta análise permite observar que as empresas sustentáveis tem na média um maior gerenciamento de resultados que as quase sustentáveis e as não sustentáveis, o que contraria os seguintes autores: Bona-Sánchez, Pérez-Alemaán, Santana-Martin (2017), Yip, Stade e Cahan (2011), Chih, Shen e Kang (2008) e Kim, Park e Wier (2012). Ademais, se nota que empresas com boas práticas de sustentabilidade mostram ter na média maior concentração acionaria e menores resultados negativos que as outras empresas. Também os dados apresentam evidencias da existência de outliers que foram retirados pelos resíduos anormais na rodagem das regressões.

As Tabelas 4 e 5 apresentam os resultados da matriz de correlação. Se pode ver entre COM e IGC, ISE e IGC e entre RES e GRIV um alto e significante coeficiente de correlação, o que indicaria uma aparente associação entre as variáveis. Porém como ISE e IGC são variáveis dummy o que se indica na matriz é uma medida de posicionamento entre 1 e 0 e não tanto uma medida de associação de variáveis. 


\section{Tabela 3 - Resultados descritivos}

\begin{tabular}{llllll}
\multicolumn{2}{l}{ Empresas não sustentáveis } & & & \\
Variável & Observações & Média & $\begin{array}{l}\text { Desvio } \\
\text { padrão. }\end{array}$ & Mínimo & Máximo \\
\hline GRIV & 4029 & 0.0487 & 0.0799 & 0.0000 & 0.9023 \\
IGC & 4271 & 0.1227 & 0.3281 & 0.0000 & 1.0000 \\
ROA & 2196 & -73.7575 & 1598.4620 & -67009.4400 & 792.4652 \\
TAM & 2200 & $1.01 \mathrm{e}+10$ & $3.69 \mathrm{e}+10$ & 2827.5130 & $8.29 \mathrm{e}+11$ \\
END & 1907 & 0.9657 & 4.1781 & 0.0000 & 117.4380 \\
CON & 2088 & 57.9802 & 28.7230 & 0.1388 & 100.0000 \\
RES & 2196 & 0.3461 & 0.4758 & 0.0000 & 1.0000 \\
MTB & 1907 & 19656.4800 & 858247.4000 & 0.0000 & $3.75 \mathrm{e}+07$ \\
\hline
\end{tabular}

Empresas quase sustentáveis

\begin{tabular}{llllll} 
Variável & Observações & Média & $\begin{array}{l}\text { Desvio } \\
\text { padrão. }\end{array}$ & Mínimo & Máximo \\
\hline GRIV & 808 & 0.0515 & 0.0752 & 0.0000 & 0.6465 \\
IGC & 850 & 0.2953 & 0.4564 & 0.0000 & 1.0000 \\
ROA & 572 & 87.9464 & 2524.2440 & -10018.1300 & 58970.8300 \\
TAM & 573 & $1.92 \mathrm{e}+10$ & $1.05 \mathrm{e}+11$ & 24861.6200 & $1.02 \mathrm{e}+12$ \\
END & 508 & 1.4605 & 8.15563 & 0.0000 & 106.6740 \\
CON & 567 & 48.8403 & 27.5137 & 3.5399 & 100.0000 \\
RES & 572 & 0.3936 & 0.4889 & 0.0000 & 1.0000 \\
MTB & 508 & 103.8665 & 2294.0150 & 0.0000 & 51706.4300 \\
\hline
\end{tabular}

Empresas sustentáveis

\begin{tabular}{llllll} 
Variável & Observações & Média & $\begin{array}{l}\text { Desvio } \\
\text { padrão. }\end{array}$ & Mínimo & Máximo \\
\hline GRIV & 219 & 0.0590 & 0.0772 & 0.0000 & 0.4464 \\
IGC & 231 & 0.2121 & 0.4097 & 0.0000 & 1.0000 \\
ROA & 149 & 2.8880 & 10.1522 & -51.7836 & 23.0937 \\
TAM & 149 & $1.68 \mathrm{e}+10$ & $2.38 \mathrm{e}+10$ & $7.63 \mathrm{e}+07$ & $1.29 \mathrm{e}+11$ \\
END & 148 & 1.1327 & 1.5515 & 0.0000 & 12.54341 \\
CON & 147 & 56.9518 & 29.0614 & 11.4116 & 100.0000 \\
RES & 149 & 0.2483 & 0.4335 & 0.0000 & 1.0000 \\
MTB & 148 & 2.9474 & 5.0227 & 0.0000 & 42.0670 \\
\hline
\end{tabular}

Nota . Significado das siglas: GRIV $=$ Gerenciamento de Resultados ISE $=$ Sustentabilidade; IGC = Governança Corporativa; ROA = Rentabilidade; TAM = Tamanho; END = Endividamento; $\mathrm{COM}=$ Concentração Acionária; RES = Resultados Negativos; $\mathrm{MTB}=$ Market-to-book . Fonte: Resultados da pesquisa. 
Tabela 4 - Resultados da matriz de correlação - Parte 1

\begin{tabular}{llllll}
\hline Variáveis & GRIV & ISE & IGC & ROA & TAM \\
\hline GRIV & 1.0000 & & & & \\
ISE & 0.0276 & 1.0000 & & & \\
IGC & -0.0095 & $0.1461^{* * *}$ & 1.0000 & & \\
ROA & $-0.0486^{* * *}$ & 0.0291 & 0.0154 & 1.0000 & \\
TAM & 0.0194 & 0.0581 & -0.0232 & 0.0049 & 1.0000 \\
END & -0.0124 & 0.0283 & 0.0013 & 0.0003 & 0.0005 \\
COM & $0.0610^{* * *}$ & $-0.0848^{* * *}$ & $-0.4504^{* * *}$ & -0.0291 & $-0.0497^{* * *}$ \\
RES & $0.2098^{* * *}$ & -0.0075 & $-0.0788^{* * *}$ & $-0.0630^{* * *}$ & -0.0302 \\
MTB & -0.008 & -0.0107 & -0.0131 & 0.0000 & -0.0045 \\
ANO & 0.0108 & $0.0688^{* * *}$ & $0.0670^{* * *}$ & -0.0003 & -0.0046
\end{tabular}

Nota. Significado das siglas: GRIV = Gerenciamento de Resultados ISE = Sustentabilidade; IGC = Governança Corporativa; ROA = Rentabilidade; TAM = Tamanho; END = Endividamento; COM = Concentração Acionária; RES = Resultados Negativos; MTB $=$ Market-to-book. Os asteriscos significam o nível de significância, ${ }^{*}$ corresponde a $10 \%,{ }^{* *}$ a $5 \%$ e *** a $1 \%$. Fonte: Resultados da pesquisa.

Tabela 5 - Análise de correlação - Parte 2

\begin{tabular}{llllll}
\hline Variáveis & END & CON & RES & MTB & ANO \\
\hline END & 1.0000 & & & & \\
CON & -0.0230 & 1.0000 & & & \\
RES & $0.0911^{* * *}$ & 0.0769 & 1.0000 & & \\
MTB & -0.0041 & 0.0341 & -0.0122 & 1.0000 & \\
ANO & -0.000 & -0.0153 & $0.0825^{* * *}$ & -0.0119 & 1.0000
\end{tabular}

Nota. Significado das siglas: GRIV $=$ Gerenciamento de Resultados ISE $=$ Sustentabilidade; IGC = Governança Corporativa; ROA = Rentabilidade; TAM = Tamanho; END = Endividamento; $\mathrm{CON}$ = Concentração Acionária; RES = Resultados Negativos; MTB = Market-to-book. Os asteriscos significam o nível de significância, ${ }^{*}$ corresponde a $10 \%,{ }^{* *}$ a $5 \%$ e ${ }^{* * *}$ a $1 \%$. Fonte: Resultados da pesquisa

Foi realizado o teste Variance Inflation Factor (VIF) para testar estatisticamente a existência de multicolinearidade entre as variáveis e para ver se as relações de associação apresentadas na matriz de correlação persistem. Estes resultados são apresentados na Tabela 6 a seguir: 
Tabela 6 - Análise de multicolinearidade

\begin{tabular}{lll}
\hline Variable & VIF & $1 /$ VIF \\
\hline ISE-1 & 1.1200 & 0.8966 \\
ISE-2 & 1.0200 & 0.9790 \\
IGC & 1.3300 & 0.7526 \\
ROA & 1.0100 & 0.9892 \\
TAM & 1.0900 & 0.9150 \\
END & 1.0200 & 0.9836 \\
CON & 1.2800 & 0.7745 \\
RES & 1.0400 & 0.9595 \\
MTB & 1.0100 & 0.9914 \\
SET-Primário & 5.3400 & 0.1871 \\
SET-Terciário & 5.2300 & 0.1913 \\
ANO-2011 & 1.8600 & 0.5451 \\
ANO-2012 & 1.8600 & 0.5450 \\
ANO-2013 & 1.8400 & 0.5558 \\
ANO-2014 & 1.8200 & 0.5605 \\
ANO-2016 & 1.8000 & 0.5616 \\
ANO-2017 & 1.7900 & 0.5637 \\
\hline Media do VIF & 1.8500 & \\
\hline
\end{tabular}

Nota . Significado das siglas: GRIV = Gerenciamento de Resultados ISE $=$ Sustentabilidade; IGC $=$ Governança Corporativa; $\mathrm{ROA}=$ Rentabili dade; $\mathrm{TAM}=$ Tamanho; $\mathrm{END}=$ Endividamento; $\mathrm{COM}=$ Concentração Acionária; RES = Resultados Negativos; MTB = Market-to-book. Fonte: Resultados da pesquisa.

Estes resultados indicam que as associações apresentadas na matriz de correlação não persistiram na análise VIF e, portanto, não foi excluída nenhuma variável do modelo.

\subsection{Análise Estatística}

Foram realizados os testes de Breusch-Pagan, Chow e Hausman onde se determinou que o tipo de regressão mais adequado aos dados é o modelo de efeitos fixos. O teste de Wald revelou que há heterocedasticidade nos dados, portanto foram utilizados os erros robustos de White (comando vce robust) no software Stata 13® para tratar essa particularidade dos dados. Em relação à auto correlação, 
depois de aplicado o teste de Wooldridge se identificou que não se apresenta este fenômeno no modelo. Finalmente em relação ao teste de normalidade de Shapiro Wilk foi evidenciada a ausência de normalidade nos dados. Alem disso, o teste $\mathrm{F}$ mostra que no conjunto as variáveis explicam bem o modelo. Foram eliminados os resíduos que eram outliers para a rodagem da regressão, este tratamento eliminou o setor primário da amostra e por isto só se apresenta uma categoria nos resultados. A seguir se apresenta a Tabela 7 com os resultados da regressão em painel de efeitos fixos.

Tabela 7 - Resultados Modelo GR segundo a Métrica de Leuz, Nanda e Wysocki (2003)

\begin{tabular}{|c|c|c|c|c|c|c|}
\hline \multirow{2}{*}{$\begin{array}{l}\text { Variáveis } \\
\text { ISE } 1\end{array}$} & \multirow{2}{*}{$\begin{array}{l}\text { Coeficiente } \\
-0.0017\end{array}$} & \multirow{2}{*}{$\begin{array}{l}\begin{array}{l}\text { Erro } \\
\text { padrão }\end{array} \\
0.0020\end{array}$} & \multirow{2}{*}{$\begin{array}{l}Z \\
-0.8500\end{array}$} & \multirow{2}{*}{$\begin{array}{l}P>Z \\
0.3950\end{array}$} & \multicolumn{2}{|c|}{$\begin{array}{l}\text { [95\% Intervalo } \\
\text { de confiança] }\end{array}$} \\
\hline & & & & & -0.0057 & 0.0022 \\
\hline ISE 2 & -0.0032 & 0.0042 & -0.7700 & 0.4410 & -0.0116 & 0.0050 \\
\hline IGC & -0.0031 & 0.0028 & -1.0700 & 0.2830 & -0.0087 & 0.0025 \\
\hline ROA & 0.0000 & 0.0000 & 1.6100 & 0.1070 & $-9.81 e-06$ & 0.0000 \\
\hline TAM & -0.0023 & 0.0009 & -2.5100 & 0.0120 & -0.0042 & -0.0005 \\
\hline END & -0.0000 & 0.0001 & -0.1600 & 0.8750 & -0.0002 & 0.0002 \\
\hline $\mathrm{CON}$ & -0.0000 & 0.0000 & -0.9800 & 0.3280 & -0.0001 & 0.0000 \\
\hline RES & 0.0017 & 0.0013 & 1.2600 & 0.2080 & -0.0009 & 0.0044 \\
\hline МТВ & $3.95 e-07$ & $5.95 \mathrm{e}-07$ & 0.6600 & 0.5070 & $-7.71 \mathrm{e}-07$ & $\begin{array}{l}1.56 \mathrm{e}- \\
06\end{array}$ \\
\hline $\begin{array}{l}\text { SET- } \\
\text { Terciário }\end{array}$ & -0.0057 & 0.0068 & -0.8600 & 0.3920 & -0.0190 & 0.0074 \\
\hline \multicolumn{2}{|l|}{$\mathrm{N}$} & 2180 & \multicolumn{2}{|c|}{$\begin{array}{l}\text { Teste de } \\
\text { Wooldridge }\end{array}$} & \multicolumn{2}{|l|}{0.5687} \\
\hline \multicolumn{2}{|l|}{$\mathrm{R}$} & 0.0339 & \multicolumn{2}{|c|}{ Teste de Wald } & \multicolumn{2}{|l|}{0.0000} \\
\hline \multicolumn{2}{|c|}{$\begin{array}{l}\text { Teste de Breusch- } \\
\text {-Pagan }\end{array}$} & 0.0000 & \multicolumn{2}{|c|}{$\begin{array}{l}\text { Teste de } \\
\text { Shapiro Wilk }\end{array}$} & \multicolumn{2}{|l|}{0.0086} \\
\hline \multicolumn{2}{|c|}{ Teste de Chow } & 0.0000 & \multirow{3}{*}{\multicolumn{2}{|c|}{$\begin{array}{l}\text { Teste F } \\
\text { Controlado } \\
\text { por Ano } \\
\text { Controlado } \\
\text { por Setor }\end{array}$}} & 0.0000 & \\
\hline \multirow{2}{*}{\multicolumn{2}{|c|}{$\begin{array}{l}\text { Teste de Hausman } \\
\text { Modelo de Dados em } \\
\text { Painel }\end{array}$}} & 0.0000 & & & \multicolumn{2}{|l|}{ Sim } \\
\hline & & Fixo & & & Sim & \\
\hline
\end{tabular}

Nota . Significado das siglas: ISE $=$ Sustentabilidade; IGC = Governança Corporativa; $\mathrm{ROA}=$ Rentabilidade; $\mathrm{TAM}=$ Tamanho; $\mathrm{END}=$ Endividamento; $\mathrm{COM}=$ Concentração Acionária; RES = Resultados Negativos; $\mathrm{MTB}=$ Market-to-book. Fonte: Resultados da pesquisa. 
A Tabela 7 mostra que não existe relação estatística significante entre Gerenciamento de Resultados (GRVI) e Sustentabilidade (ISE). Portanto, a hipótese da pesquisa não pôde ser comprovada. Isto quer dizer que a sustentabilidade não afeta o gerenciamento de resultados das empresas, o que contraria os achados de Bona-Sánchez, Pérez-Alemaán, Santana-Martin (2017), Yip, Stade e Cahan (2011), Chih, Shen e Kang (2008) e Kim, Park e Wier (2012).

No que tange às variáveis de controle, somente o tamanho (TAM) apresentou significância estatística no modelo. O tamanho da empresa revelou uma relação inversa com o gerenciamento de resultados a uma significância de $5 \%$, o que indica que quanto maior for a empresa, menor será o gerenciamento de resultados. O TAM é uma variável amplamente utilizada para explicar o gerenciamento de resultados e utilizada em diversas pesquisas como de Barros, Soares e Lima (2013), Consoni, Colauto e Lima (2017), Cunha e Piccoli (2017), Dechow, Ge e Schrand (2010), Nardi e Nakao (2009), Rathke et al (2016), Reis, Lamounier e Bessan (2015), Silva (2017) e Sprenger, Kronbauer e Costa (2017). Porém os trabalhos divergem quanto aos resultados para esta variável. No caso desta pesquisa, se infere que quando as empresas são maiores, elas são capazes de implementar melhores controles internos, auditorias, fiscalização e melhores sistemas, o que pode diminuir a incidência de gerenciamento de resultados.

\subsection{Teste de Robustez}

Foi realizado um teste de robustez para avaliar o modelo proposto. A variável de Gerenciamento de Resultados foi calculada neste teste pela metodologia de Kang e Sivaramakrishnan (1995). Tal métrica é amplamente utilizada no mundo e no Brasil, tendo sido explicada nos estudos de Martinez (2008). Ademais, outros estudos que também a adotam são: Almeida e Cunha (2017); Barros, Soares e Lima (2013); Erfurth, Bezerra (2013); Nardi e Nakao (2009); Santos e Barros (2011); Silva (2017).

Nesta metodologia, os accruals discricionários são calculados por meio dos resíduos de uma regressão com variáveis instrumentais. Além disso, são feitos três modelos para enxergar a grandeza do gerenciamento de resultados (que é tido como o valor absoluto 
das accruals discricionários) e a sua direção (accruals discricionários negativos e positivos) como realizado por Silva (2017). O teste de Hausman indicou que o tipo de regressão que melhor se adapta aos dados foi o dos efeitos aleatórios. Além disso, nos modelos a seguir persiste a não normalidade e a heterocesdasticidade nos dados e diferentemente do modelo anterior, neste está presente a autocorrelação. $\mathrm{O}$ conjunto das variáveis se apresentam influentes no modelo segundo o teste F Os resultados do teste se apresentam na Tabela 8 a seguir:

Tabela 8 - Resultados do modelo Kang e Sivaramakrishnan (1995)

\begin{tabular}{llll}
\hline Variáveis & $\begin{array}{l}\text { Accruals } \\
\text { Absolutos (1) }\end{array}$ & $\begin{array}{l}\text { Accruals } \\
\text { Positivos (2) }\end{array}$ & $\begin{array}{l}\text { Accruals } \\
\text { Negativos (3) }\end{array}$ \\
\hline ISE-1 & -0.0087 & -0.0023 & 0.0026 \\
ISE-2 & -0.0046 & -0.0059 & 0.0026 \\
IGC & -0.0030 & 0.0098 & 0.0050 \\
ROA & 0.0000 & -0.0000 & $-0.0002^{*}$ \\
TAM & -0.0005 & $-0.0012^{*}$ & 0.0000 \\
END & 0.0001 & -0.0000 & 0.0001 \\
Com & 0.0000 & 0.0002 & 0.0000 \\
RES & 0.0022 & -0.0024 & -0.0031 \\
MTB & 0.0000 & 0.0000 & -0.0002 \\
SET- Terciário & $0.0086^{* *}$ & 0.0051 & $-0.0129 * *$ \\
\hline N & 2172.0000 & 854.0000 & 1318.0000 \\
RMSE & 0.0407 & 0.0564 & 0.0267 \\
Teste Breush-Pagan & 0.0000 & 1.0000 & 0.0000 \\
Teste Chow & 0.0000 & 0.8782 & 0.0000 \\
Teste Hausman & 0.2668 & 0.5629 & 0.1365 \\
Teste Wooldrige & 0.0000 & 0.0000 & 0.0000 \\
Teste Wald & 0.0000 & 0.0000 & 0.0000 \\
Teste Shapiro Wilk & 0.0000 & 0.0000 & 0.0000 \\
Teste F & 0.0000 & 0.0000 & 0.0092 \\
\hline
\end{tabular}

Nota . Significado das siglas: ISE $=$ Sustentabilidade; IGC = Governança Corporativa; $\mathrm{ROA}=$ Rentabilidade; TAM = Tamanho; $\mathrm{END}=$ Endividamento; $\mathrm{COM}=$ Concentração Acionária; RES = Resultados Negativos; MTB $=$ Market-to-book. Os asteriscos significam o nível de significância, ${ }^{*}$ corresponde a $10 \%,{ }^{* *}$ a $5 \%$ e ${ }^{* *}$ a $1 \%$ Fonte: Resultados da pesquisa.

O modelo 1 da Tabela 8 mostra que nenhuma das variáveis do estudo (com exceção do setor) tem relação significante com o 
gerenciamento de resultados. Para o modelo 2, observou-se uma relação negativa do GRIV com a variável tamanho (TAM), ou seja, empresas maiores apresentam menor probabilidade de gerenciar resultados para aumenta-los. Estes achados corroboram os estudos de Barros, Soares e Lima (2013), Consoni, Colauto e Lima (2017), Cunha e Piccoli (2017), Dechow, Ge e Schrand (2010), Nardi e Nakao (2009), Rathke et al (2016), Reis, Lamounier e Bessan (2015), Silva (2017) e Sprenger, Kronbauer e Costa (2017). Também esta relação inversa de tamanho com o gerenciamento de resultados concorda com os achados da regressão feita na seção anterior com o modelo Leuz, Nanda e Wysocki (2003).

Por sua vez, para o modelo 3 que mede o quanto as empresas gerenciam os seus resultados visando diminuí-los, as únicas variáveis que apresentaram significância estatística foram a rentabilidade sobre o ativo (ROA) e o setor (SET). Isto quer dizer que empresas com maior performance medida pelo ROA terão menor probabilidade de gerenciar resultados para diminuí-los. Este achado é consistente com os trabalhos de Almeida, Cunha (2017), Barros, Soares e Lima (2013;); Consoni, Colauto e Lima (2017); Cuadrado-Ballesteros, Martínez-Ferrero, García-Sánchez (2017); Nardi, Nakao (2009); Rathke et al. (2016); Reis, Lamounier e Bessan, (2015); Sprenger, Kronbauer e Costa (2017).

O setor (SET) apresentou significância estatística no modelo dos accruals absolutos e no dos accruals negativos, indicando que há mudanças significativas entre as categorias setor segundo o gerenciamento de resultados. Este achado indica que as empresas do setor terciário tendem a gerenciar mais os seus resultados e tendem a gerenciar menos seus resultados para baixo (accruals negativos), quando se toma as empresas do setor secundário como base.

\section{CONSIDERAÇõeS FINAIS}

Este estudo buscou analisar a relação entre sustentabilidade empresarial e gerenciamento de resultados para firmas brasileiras de capital aberto no período de 2010 a 2017. A amostra final foi composta por 673 empresas que foram classificadas em sustentáveis, elegíveis à sustentáveis e não sustentáveis. O método de análise envolveu regressão com dados em painel, adotando o modelo de efeitos fixos. 
Adotou-se como variável dependente o gerenciamento de resultados calculado por meio de duas metodologias: a de Leuz, Nanda e Wysocki (2003) e a de Kang e Sivaramakrishnah (1995) e, como variável independente de teste, a sustentabilidade empresarial, medida pelo ISE da B3. Também foram inseridas variáveis de controle relacionadas às características das firmas.

Os resultados da presente pesquisa apontam que não há relação entre sustentabilidade empresarial (ISE) e gerenciamento de resultados (GRIV), portanto a hipótese do trabalho não pôde ser comprovada (Empresas pertencentes ao ISE gerenciam menos os resultados), contrariando em parte os resultados de Bona-Sánchez, Pérez-Alemaán, Santana-Martin (2017), Yip, Stade e Cahan (2011), Chih, Shen e Kang (2008) e Kim, Park e Wier (2012). A variável que apresentou significância no modelo foi a variável de controle correspondente ao tamanho (TAM) que teve uma relação inversa e significante com o gerenciamento de resultados (GRI).

Além disso, foi feito um teste de robustez utilizando como variável de gerenciamento de resultados a proxy de Kang e Sivaramakrishnan (1995). Este teste mostrou que não há relação estatística entre GR e Sustentabilidade e obteve também como resultado que TAM tem uma relação inversa com o gerenciamento positivo de resultados e que ROA tem uma relação também inversa com o gerenciamento negativo de resultados. Os dados correspondentes ao TAM e GR foram semelhantes e congruentes com os resultados obtidos utilizando o modelo de Leuz, Nanda e Wysocki (2003).

Uma possível justificativa para a sustentabilidade empresarial não influenciar no nível de gerenciamento de resultados pode envolver algumas deficiências das metodologias de divulgação de sustentabilidade (como o ISE), que acabam manipulando a percepção dos acionistas e escondendo algumas práticas corporativas - o chamado "green washing" - e a falta de atenção na avaliação do desempenho sustentável (SIEW, 2015).

Este trabalho trata de uma temática pouco investigada na literatura de finanças - sustentabilidade versus gerenciamento de resultados. No Brasil, não foi encontrado nenhum estudo sobre esta relação e mesmo no exterior os estudos são escassos, o que mostra a necessidade de explorar mais o assunto. Além disso, houve utili- 
zação de novas variáveis nos modelos econométricos que acrescentam uma contribuição metodológica à discussão do tema, como por exemplo, a variável Resultados Negativos, pouco utilizada no Brasil.

Também pela importância que tem o estudo do gerenciamento de resultados no Brasil e em outros países da América Latina, onde este tipo de prática é muito comum e prejudicial, o ideal é estudar a respeito para formular soluções. Como principais limitantes do trabalho se destaca a desproporcionalidade do número de empresas sustentáveis com as não sustentáveis. Para trabalhos futuros se recomenda mensurar o gerenciamento de resultados, a sustentabilidade e/ou a governança corporativa com outras proxies.

\section{REFERÊNCIAS}

ALMEIDA, A.; CUNHA, D. Estudo do mercado brasileiro de renda fixa e o perfil do investidor brasileiro. [s.l.] Universidade Federal do Rio de Janeiro, 2017.

AMEER, R.; OTHMAN, R. Sustainability practices and corporate financial performance: A study based on the top global corporations. Journal of Business Ethics, v. 108, n. 1, p. 61-79, 2012.

B3. Índice de sustentabilidade empresarial ( ISE ) índice de sustentabilidade empresarial ( ISE ). Disponível em: <http://www.bmfbovespa.com.br/pt_br/produtos/indices/indices-de-sustentabilidade/indice-de-sustentabilidade-empresarial-ise.htm>.

BARROS, C.; SOARES, R.; LIMA, G. A Relação Entre Governança Corporativa e Gerenciamento de Resultados Em Empresas Brasileiras. Revista de Contabilidade e Organizações, v. 19, n. 1976, p. 27-39, 2013.

BHASA, M. P. Global corporate governance: debates and challenges. Corporate Governance: The international journal of business in society, v. 4, n. 2, p. 5-17, 2004.

BM\&FBOVESPA. Metodologia do índice de sustentabilidade. São Paulo: [s.n.]. Disponível em: <http://www.bmfbovespa.com.br/pt_br/produtos/indices/indices-de-sustentabilidade/ indice-de-sustentabilidade-empresarial-ise.htm>.

BONA-SÁNCHEZ, C.; PÉREZ-ALEMÁN, J.; SANTANA-MARTIN, D. J. Sustainability disclosure, dominant owners and earnings informativeness. Research in International Business and Finance, v. 39, p. 625-639, 2017.

CALDAS, C. B.; TAMBOSI FILHO, E.; VIEIRA, A. M. Governança Corporativa e Sustentabilidade: uma relação necessária. Revista UNIABEU, v. 7, p. 353-369, 2014.

CHIH, H.; SHEN, C.; KANG, F. Corporate Social Responsibility , Investor Protection , and Earnings Management : Some International Evidence. Journal of Business Ethics, v. 79, n. 179-198, p. 179-198, 2008.

CONSONI, S.; COLAUTO, R. D.; LIMA, G. A. S. F. DE. A divulgação voluntária e o gerenciamento de resultados contábeis : evidências no mercado de capitais brasileiro. Revista Contabilidade \& Finanças-USP, v. 28, n. 74, p. 249-263, 2017. 
CUADRADO-BALLESTEROS, B.; MARTÍNEZ-FERRERO, J.; GARCÍA-SÁNCHEZ, I. M. Mitigating information asymmetry through sustainability assurance: The role of accountants and levels of assurance. International Business Review, v. 26, n. 6, p. 1141-1156, 2017.

CUNHA, P. R. DA; PICCOLI, M. R. Influência do board interlocking no gerenciamento de resultados. Revista Contabilidade \& Finanças, v. 28, n. 74, p. 179-196, 2017.

DECHOW, P.; GE, W.; SCHRAND, C. Understanding earnings quality: A review of the proxies, their determinants and their consequences. Journal of Accounting and Economics, v. 50, n. 2-3, p. 344-401, 2010.

ECCLES, R. G.; IOANNOU, I.; SERAFEIM, G. The impact of corporate sustainability on organizational processes and performance. Management Science, v. 60, n. November, p. 2835-2857, 2014.

ELKINGTON, J. Sustentabilidade. Canibais com Garfo e Faca. [s.l.] Makron Books, 2001.

ERFURTH, A. E.; BEZERRA, F. A. Gerenciamento de resultados nos diferentes níveis de governança corporativa. Revista de Administração e Contabilidade da Unisinos, v. 10, n. 1, p. 32-42, 2013.

GOYAL, P.; RAHMAN, Z.; KAZMI, A. A. Corporate sustainability performance and firm performance research literature review and future research agenda. Management Decision, v. 51, n. 2, p. 361-379, 2013.

GUIMARÃES, T. M.; PEIXOTO, F. M.; CARVALHO, L. Sustentabilidade empresarial e governança corporativa : uma análise da relação do ise da BM\&FBovespa com a compensação dos gestores de empresas brasileiras. Revista de Educação e Pesquisa em Contabilidade, v. 11, n. 2, p. 134-149, 2017.

HOLANDA, A.; CARLOS, A.; COELHO, D. Gerenciamento de Resultados e Estrutura de Propriedade : Evidências no Brasil. IV Congresso Nacional de Administração e Ciências Contábeis - AdCont 2013. Anais...2013

IBRAHIM, M. S. et al. Analysis of earnings management practices and sustainability reporting for corporations that offer islamic products \& services. Procedia Economics and Finance, v. 28, n. April, p. 176-182, 2015.

KANG, S.-H. A Conceptual and Empirical Evaluation of Accrual Prediction Models. [s.l.] Yale University, 1999.

KANG, S.-H.; SIVARAMAKRISHNAN, K. Issues in Testing Earnings Management and an Instrumental Variable Approach. Journal of Accounting Research, v. 33, n. 2, p. 353, 1995.

KANTABUTRA, S. Predicting corporate sustainability: A Thai approach. Journal of Applied Business Research, v. 27, n. 6, p. 123-133, 2011.

KHAN, M.; SERAFEIM, G.; YOON, A. Corporate Sustainability : First Evidence on Materiality. SSRN Electronic Journal, p. 1-55, 2015.

KIM, Y.; PARK, M. S.; WIER, B. Is earnings quality associated with corporate social responsibility? The Accounting Review, v. 87, n. 3, p. 761-796, 2012.

KRECHOVSKÁ, M.; PROCHÁZKOVÁ, P. T. Sustainability and its integration into corporate governance focusing on corporate performance management and reporting. Procedia Engineering, v. 69, p. 1144-1151, 2014. 
LAMEIRA, V. DE J. et al. Sustentabilidade, valor , desempenho e risco no mercado de capitais brasileiro sustainability, value, performance and risk in the brazilian capital markets. Revista Brasileira de Gestão de Negocios, v. 15, n. 46, p. 76-90, 2013.

LEHMANN, N. The role of corporate governance in shaping accruals manipulation prior to acquisitions The role of corporate governance in shaping accruals manipulation prior to acquisitions. Accounting and Business Research, v. 4788, n. March, 2016.

LEUZ, C.; NANDA, D.; WYSOCKI, P. D. Earnings management and investor protection : an international comparison. Journal of Financial Economics, p. 0-33, 2003.

MARTINEZ, A. L. Detectando earnings management no brasil : estimando os accruals discricionários. Revista Contabilidade \& Finanças-USP, v. 19, n. 49, p. 7-17, 2008.

MARTINEZ, A. L.; MORAES, A. DE J. Relationship between auditors' fees and earnings management. Revista de Administração de Empresas, v. 57, n. 2, p. 148-157, 2017.

MARTINS, M. N. DA S. P.; CAMPOS, A. L. S.; MARTINS, F. S. Influência dos investimentos sociais para inclusão de empresas no índice de sustentabilidade empresarial (ISE). RGSA Revista de Gestão Social e Ambiental, v. 10, p. 58-71, 2016.

NARDI, P. C. C.; NAKAO, S. H. Gerenciamento de resultados e a relação com o custo da dívida das empresas brasileiras abertas. Revista Contabilidade \& Finanças-USP Contabilidade, v. 20, n. 50, p. 77-100, 2009.

NUNES, J. G. et al. A adesão das empresas ao índice BM\&FBovespa de sustentabilidade empresarial. BASE - Revista de Administração e Contabilidade da Unisinos, v. 7, n. 4, p. 328-340, 2010.

PEIXOTO, F. M. et al. Custo de capital, endividamento e sustentabilidade empresarial: um estudo no mercado de capitais brasileiro no período de 2009 a 2013. RACE-Revista de Administração, Contabilidade e Economia, v. 15, n. 1, p. 39-66, 2016.

PRIOR, D.; SURROCA, J.; TRIBÓ, J. A. Earnings Management and Corporate Social Responsibility. Business Economics Series, v. 06, p. 06-23, 2007.

RAHMAWATI; DIANITA, P. Analysis of the effect of corporate social responsibility on financial performance with earnings management as a moderating variable. Journal of Modern Accounting and Auditing, v. 7, n. 10, p. 1034-1045, 2011.

RATHKE, A. A. T. et al. International financial reporting standards and earnings management in latin america. Revista de Administração Contemporânea, v. 20, n. 3, p. 368-388, 2016.

REIS, E. M. DOS; LAMOUNIER, W. M.; BESSAN, V. G. F. Evitar divulgar perdas : um estudo empírico do gerenciamento de resultados por meio de decisões operacionais. Revista Contabilidade \& Finanças-USP, v. 26, n. 69, p. 247-260, 2015.

REZAEE, Z.; TUO, L. Voluntary disclosure of non-financial information and its association with sustainability performance. Advances in Accounting, v. 39, n. May 2016, p. 47-59, 2017.

SANDRIN, R. A.; SOARES, R. O. Gerenciamento de resultados en empresas latino-americanas com adr's negociados nos Estados Unidos. Revista de contabilidade e controladoria, n. 2003, p. 113-127, 2012.

SANTOS, J.; BARROS, C. O que determina a tomada de decisão financeira: razão ou emoção?

Revista Brasileira de Gestão de Negócios, v. 13, n. 38, p. 663-679, 2011. 
SIEW, R. Y. J. A review of corporate sustainability reporting tools ( SRTs ). Journal of Environmental Management, v. 164, p. 180-195, 2015.

SILVA, N. D. Fatores determinantes do gerenciamento de resultados em estatais brasileiras. [s.1.] Universidade Federal de Uberlândia, 2017.

SILVA, R. L. M. Adoção completa das IDRS no Brasil: Qualidade das demostrações contábeis e o custo de capital próprio. [s.l.] Universidade de São Paulo, 2013.

SPRENGER, K. B.; KRONBAUER, C. A.; COSTA, C. M. Características do CEO e o gerenciamento de resultados em empresas listadas na BM\&FBovespa. Revista Universo Contábil, v. 13, n. 3, p. 120-142, 2017.

WEBER, O. Corporate sustainability and financial performance of Chinese banks. Sustainability Accounting, Management and Policy Journal, v. 8, n. 3, p. 358-385, 2017.

YIP, E.; STADE, C. VAN; CAHAN, S. Corporate social responsibility reporting and earnings management: the role of political costs. Australasian Accounting Business \& Finance Journal, v. 5, n. 3, p. 17-34, 2011.

Recebido em: 5-8-2019

Aprovado em: 17-11-2020

Avaliado pelo sistema double blind review.

Disponível em http://mjs.metodista.br/index.php/roc 